\title{
Serum circulating microRNA profiling for identification of potential breast cancer biomarkers
}

\author{
Fermín Mar-Aguilar $^{\mathrm{a}}$, Jorge A. Mendoza-Ramírez ${ }^{\mathrm{a}}$, Ismael Malagón-Santiago ${ }^{\mathrm{a}}$, Perla K. Espino-Silva ${ }^{\mathrm{d}}$, \\ Sandra K. Santuario-Facioc ${ }^{c}$ Pablo Ruiz-Flores ${ }^{\mathrm{d}}$, Cristina Rodríguez-Padilla ${ }^{\mathrm{b}}$ and \\ Diana Reséndez-Pérez ${ }^{\mathrm{a}, \mathrm{b}, *}$ \\ ${ }^{a}$ Departamento de Biología Celular y Genética, Universidad Autónoma de Nuevo León, San Nicolás de los Garza, \\ Nuevo León, México \\ ${ }^{\mathrm{b}}$ Departamento de Inmunología y Virología, Facultad de Ciencias Biológicas, Universidad Autónoma de Nuevo \\ León, San Nicolás de los Garza, Nuevo León, México \\ ${ }^{\mathrm{c}}$ Departamento de Bioquímica, Facultad de Medicina, Universidad Autónoma de Nuevo León, San Nicolás de los \\ Garza, Nuevo León, México \\ ${ }^{\mathrm{d}}$ Departamento de Genética y Medicina Molecular, Facultad de Medicina, Universidad Autónoma de Coahuila, \\ Torreón, Coahuila, México
}

\begin{abstract}
MicroRNAs (miRNAs) are a class of small, non-coding RNA molecules that can regulate gene expression, thereby affecting crucial processes in cancer development. miRNAs offer great potential as biomarkers for cancer detection because of their remarkable stability in blood and their characteristic expression in different diseases. We investigated whether quantitative RT-PCR miRNA profiling on serum could discriminate between breast cancer patients and healthy controls. We performed miRNA profiling on serum from breast cancer patients, followed by construction of ROC (Receiver Operating Characteristic) curves to determine the sensitivity and specificity of the assay. We found that seven miRNAs (miR-10b, miR-21, miR-125b, miR-145, miR-155 miR-191 and miR-382) had different expression patterns in serum of breast cancer patients compared to healthy controls. ROC curve analyses revealed that three serum miRNAs could be valuable biomarkers for distinguishing BC from normal controls. Additionally, a combination of ROC curve analyses of miR-145, miR-155 and miR-382 showed better sensitivity and specificity of our assay. miRNA profiling in serum has potential as a novel method for breast cancer detection in the Mexican population.
\end{abstract}

Keywords: Breast cancer, miRNAs, biomarkers, serum, miR-382

\section{Introduction}

Breast cancer (BC) is one of the leading causes of cancer death among women worldwide [1]. Many studies on $\mathrm{BC}$ detection and treatment have been published

*Corresponding author: Dr. Diana Reséndez-Pérez, Departamento de Biología Celular y Genética, Facultad de Ciencias Biológicas, Universidad Autónoma de Nuevo León, Av. Manuel Barragan y Pedro de Alba s/n, Cd Universitaria, San Nicolás de los Garza, Nuevo León, CP. 66451, México. Tel.: +52 8183294000 ext. 2926; Fax: +52 81 8352 4212; E-mail: diana.resendezpr@uanl.edu.mx. in recent years. Whereas most studies focus on improving prognosis with better therapies, a more promising approach is to detect cancer at an early stage. The current standard diagnostic tools, mammography and ultrasound, have been successful in the detection of early-stage BC. However, there is a need for new, minimally invasive diagnostic approaches are to supplement breast imaging and improve detection rates and BC screening compliance. Recently, the discovery of microRNAs (miRNAs), a class of small, nonproteincoding RNAs, has opened new opportunities in cancer biology. miRNAs are regulatory, nonprotein-coding, 9- 
to 25-nucleotide-long RNA molecules that regulate the expression of a variety of genes by sequence-specific base pairing on the 3' untranslated regions (3'UTR) of the target mRNA, resulting in mRNA degradation or inhibition of translation. Patterns of miRNA expression are meticulously regulated and play important roles in oncogenesis [2,3]. Some miRNAs exert negative control over the expression of numerous oncoproteins in normal cells and consequently their deregulation is believed to be an important mechanism behind cancer development and progression. Because of their distinct patterns of expression associated with cancer type, remarkable stability in blood and other body fluids, miRNAs are considered to be highly promising cancer biomarkers $[4,5]$.

The extreme stability of circulating miRNAs in the RNase-rich environment of the bloodstream is the basis of their value as biomarkers [6]. The mechanism that makes miRNAs so stable is only being understood. microRNAs are released from cells wrapped in proteins or microvesicles. Selectively exported miRNAs are packaged in structures that are different from those that carry neutral released miRNAs (n-miRNAs). Malignant cells release greater quantities of selectively exported miRNAs, whereas the release of n-miRNAs is not affected by malignancy. These results suggest that malignant transformation alters the pathways through which specific miRNAs are exported from cells. These changes in the microvesicles and their miRNA cargo could be used to detect the presence of malignant cells in the body [7]. Six miRNAs (miR-10b, miR-21, miR125b, miR-145, miR-155 and miR-191) are known to be aberrantly expressed in breast cancer tissues [8]. The identification of circulating miRNAs as potential noninvasive biomarkers for BC and other diseases was followed by initial studies trying to associate these markers with relapse-free survival, overall survival, and response to therapy [9-11]. The aim of our study was to analyze the miRNA expression patterns in serum of patients with $\mathrm{BC}$ in comparison to healthy controls using quantitative RT-PCR.

\section{Methods}

\subsection{Biological material}

Samples from patients with pathologically confirmed breast cancer were collected at the Center of Biomedic Investigation in Torreón Coahuila, México. The control samples were provided by healthy women. All par-
Table 1

Stage of breast cancer in patients

\begin{tabular}{ccc}
\hline No. patients & Stage & Range of age (years) \\
\hline 13 & I & $42-73$ mean $=54.61$ \\
14 & II & $40-68$ mean $=50.85$ \\
34 & III & $45-77$ mean $=53.09$ \\
\hline
\end{tabular}

ticipants were Mexican-born women who had given their written, informed consent to participate in this study. The Institutional Review Board of the College of Biological Sciences of the Autonomous University of Nuevo León approved the collection of serum samples in accordance with institutional protocol. A total of 61 breast cancer and 10 normal blood samples were analyzed in this study. The breast cancer tissue analyzed (Table 1) correspond to ductal carcinoma (stages I, II, and III).

\subsection{RNA extraction}

The blood samples were collected from patients before surgery. Each sample was centrifuged at 3000 RPM for 5 minutes to separate serum and then stored at $-70^{\circ} \mathrm{C}$ until RNA extraction. We isolated total RNA containing small RNA from $400 \mu \mathrm{L}$ of serum previously thawed on ice using miRNAeasy kit (Qiagen, Valencia, CA, USA) according to the manufacturer's protocol. The RNA was eluted with $50 \mathrm{~mL}$ of RNAse-free water and was stored at $-70^{\circ} \mathrm{C}$ until RT-PCR reaction. We quantified the concentration of all RNA samples using NanoDrop 1000 (Thermo Scientific, Wilmington, DE, USA).

\section{3. $\operatorname{miRNA} q R T-P C R$}

We used Taqman miRNA assays to perform expression profiling of breast-tissue miRNA. All reagents, primers, and probes were obtained from Applied Biosystems, (Foster City, CA, USA). We used 20 ng of total RNA from each sample for the individual assays in $15 \mu \mathrm{L}$ reactions containing reverse transcription (RT) mixture and primers. The mix was incubated at $16^{\circ} \mathrm{C}$ for $30 \mathrm{~min}, 42^{\circ} \mathrm{C}$ for $60 \mathrm{~min}$ and $85^{\circ} \mathrm{C}$ for 5 min. miRNA expression levels were quantified using the Mx3005P qPCR System (Agilent, Santa Clara, CA, USA). For this purpose, $1 \mu \mathrm{L}$ of RT reaction was mixed with $10 \mu \mathrm{L}$ of $2 \mathrm{X}$ Taqman PCR mixture, $1 \mu \mathrm{L}$ of Taqman primer mix and probe and $8 \mu \mathrm{L}$ water to get a final volume of $20 \mu \mathrm{L}$. An RT-PCR was done in triplicate, including non-template controls. The relative expression of the mature miRNA was calculated using the comparative cycle threshold $\left(2^{-\Delta \Delta C T}\right)$ method. We used 18S RNA as an endogenous control to normalize the data [12]. 

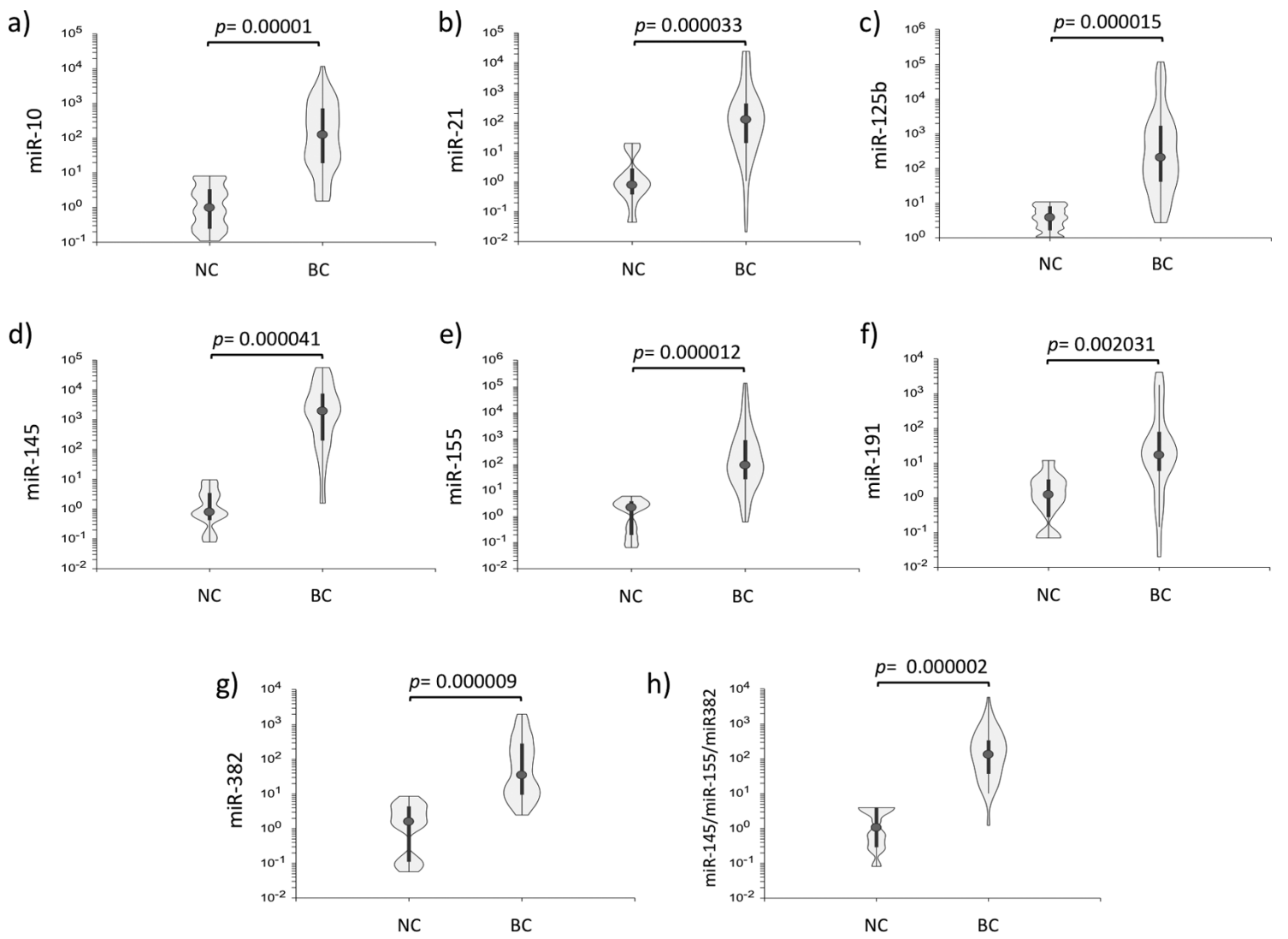

Fig. 1. Relative fold change expression levels of (a) miR-10b, (b) miR-21, (c) miR-125b, (d) miR-145, (e) miR-155, (f) miR-191, (g) miR-382 and (h) miR-145/miR-155/miR-382 in breast cancer (BC) and normal breast tissue (NC). The $p$-values for all miRNAs was significative. The dot at the center of violin plot represents the mean fold change for each miRNA.

Table 2

AUCs (area under the ROC curves)

\begin{tabular}{cccc}
\hline miRNA & AUC & Standard error & $95 \%$ CI \\
\hline miR-10b & 0.9500 & 0.03622 & 0.9137 to 0.9862 \\
miR-21 & 0.9500 & 0.03732 & 0.9126 to 0.9873 \\
miR-125b & 0.9500 & 0.03626 & 0.9137 to 0.9862 \\
miR-145 & 0.9777 & 0.02400 & 0.9537 to 1.0017 \\
miR-155 & 0.9944 & 0.00786 & 0.9866 to 1.0022 \\
miR-191 & 0.7944 & 0.08680 & 0.7076 to 0.8812 \\
miR-382 & 0.9666 & 0.02865 & 0.9379 to 0.9952 \\
\hline
\end{tabular}

\subsection{Statistical analysis}

We determined the normal distribution of the level of expression $\left(2^{-\Delta \Delta C T}\right)$ for all the miRNAs with a two-tailed D'Agostino-Pearson $\mathrm{K}^{2}$ test. We evaluated the differential expression of miRNA between breast cancer and normal samples with a two-tailed MannWhitney U test. Validation of miRNA Diagnostic Test from each miRNA and from the three selected combined miRNAs was carried-out by Receiver operating characteristic (ROC) curves analysis: area under curve (with 100 points), efficiency, diagnostic sensibility and specificity, positive/negative threshold (cutoff), positive and negative predictive values. Optimal cutoff by highest sensibility plus specificity and efficiency values were determined. ROC analysis for miRNA-combined, geometric mean of level expression was calculated by three miRNA selected with highest efficiency values. $\mathrm{P}$-values equal or lesser than 0.05 were considered statistically significant. The data analysis was done using NCSS 2007 version 07.1.20 (NCSS, LLC).

\section{Results}

To investigate whether the levels of the seven miRNAs (miR-10b, miR-21, miR-125b, miR-145, miR155, miR-191 and miR-382) presented desregulation in serum of patients with BC, we measured the serum levels of the seven miRNAs in $61 \mathrm{BC}$ patients and 10 healthy controls. 

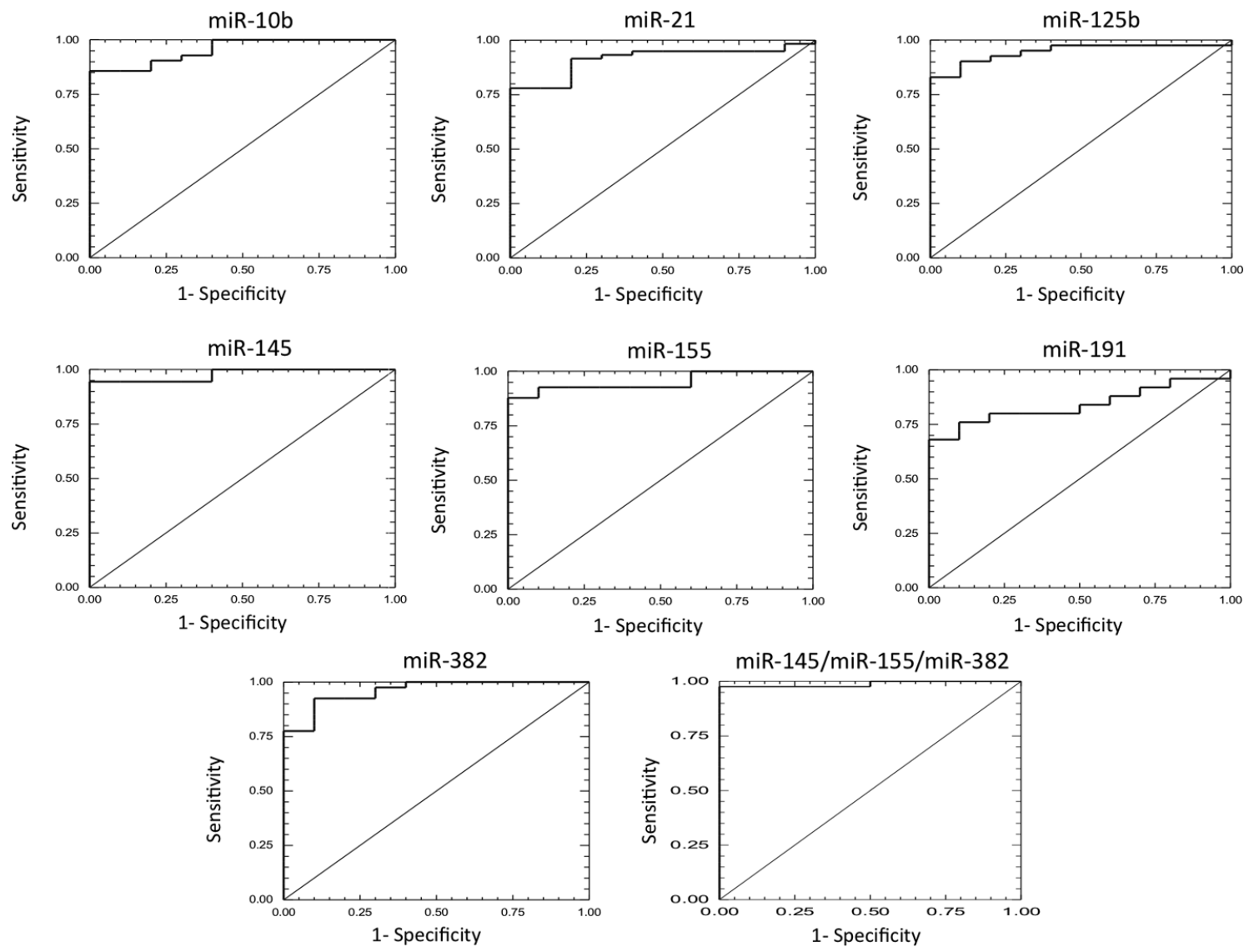

Fig. 2. Area under curve of receiver operating characteristic (ROC) for miR-10b, miR-21, miR-125b, miR-145, miR-155, miR-191, miR-382 and miR-145/miR-155/miR-382. ROC curves for each miRNA. All miRNAs shows lower sensitivity and specificity values than the combination of $\mathrm{miR}-145 / \mathrm{miR}-155 / \mathrm{miR}-382$.

As shown in Fig. 1, the concentrations of the seven miRNAs were significantly higher in $\mathrm{BC}$ serum than in healthy controls $(p<0.001)$. ROC curve analyses, performed to evaluate the diagnostic value for the seven serum miRNAs, revealed that 3 of them were valuable biomarkers for distinguishing $\mathrm{BC}$ from normal controls. The AUCs (area under the ROC curves), standard error and confidence interval (CI) for each miRNA are showed at Table 2.

At the optimal cutoff value of 59.22 with the value of sensitivity + specificity considered to be maximal for miR-10b, the sensitivity and specificity were $83.30 \%$ and $100.00 \%$; at the cutoff value of 6.48 for miR-21, the sensitivity and specificity were $94.40 \%$ and $80.00 \%$; at the cutoff of 8.46 for miR-125b, the sensitivity and specificity were $88.90 \%$ and $80.00 \%$; at the cutoff of 15.93 for miR-145, the sensitivity and specificity were $94.40 \%$ and $100.00 \%$; at the cutoff of 7.92 for miR155 , the sensitivity and specificity were $94.40 \%$ and $100.00 \%$; at the cutoff of 11.59 for miR-191, the sen- sitivity and specificity were $72.2 \%$ and $90.00 \%$; and at the cutoff of 4.85 for miR-382, the sensitivity and specificity were $94.40 \%$ and $90.00 \%$, Fig. 2 .

To elevate the diagnosis value we performed the combination ROC curve analyses of miR-145, miR-155 and miR-382. The ratio of combination for these miRNAs yielded the highest AUC value of 0.9878 (95\% CI: 0.9065 to 0.9985 ) and at the optimal cutoff value of 10.07 , the sensitivity and specificity were $97.60 \%$ and $100 \%$, Fig. 2. Finally efficiency of a diagnostic test, defined as percentage of subjects correctly classified as diseased of healthy was $85.7 \%$ for miR-10b, $89.3 \%$ for miR-21, 89.3\% for miR-125b, 96.4\% for miR-145, $96.4 \%$ for miR-155, $78.6 \%$ for miR-191, $92.6 \%$ miR382 and $98 \%$ for combination of miR-145, miR-155 and miR-382. These results indicate that the combination signature has a strong potential diagnosis value for $\mathrm{BC}$ detection.

The serum levels of miR-10b, miR-21, miR-125b, miR-145, miR-155, miR-191 and miR-382 in the BC 
patients at different TNM stages (13 with stage I, 14 with stage II, and 34 with stage III) were analyzed to determine whether the seven serum miRNAs could detect early-stage BC. The serum levels of the seven miRNAs were not significantly different across three stages.

\section{Discussion}

In this study, we analyzed the levels of seven miRNAs (miR-10b, miR-21, miR-125b, miR-145, miR155, miR-191 and miR-382) in serum from 61 BC patients and 10 healthy controls. Our assay showed significantly higher serum levels of miR-10b in BC patients in contrast with those reported by Heneghan et al. showing a level of expression of miR-10b similar to the healthy control [13]. We found that the levels of miR-21 were significantly higher in BC serum than in the controls, which are consistent with the report by Asaga et al. They found that high-circulating miR-21 concentrations correlated significantly with the presence and extent of breast cancer [14]. miR-125b was significantly over expressed in this assay, just as was reported by Wang et al., who said that miR-125b was significantly associated with therapeutic response, exhibiting higher expression level in non-responsive patients [15]. miR-145 was significantly higher in BC serum than in the controls. This miRNA has been found to be significantly elevated in malignant myoepithelioma but not in basal cell-like breast cancer [16]. miR145 is known as a tumor suppressor in numerous human cancers, exhibits an inhibitory role in tumoral angiogenesis, cell growth and invasion and tumor growth through the post-transcriptional regulation of the novel targets N-RAS and VEGF-A, and may plays important inhibitory role in breast cancer malignancy by targeting these genes [17], early manifestation of altered miR145 expression in atypical hyperplasia and carcinoma in situ lesions in BC suggests that this miRNA may have a potential clinical application as a novel biomarker for early detection [18]. We found that the levels of miR-155 were significantly higher in BC serum than in controls. Up-regulation of miR-155 has been observed in human primary breast cancer [19], and significantly over-expressed in the tumor specimens of BC. miR-155 was postulated as a potential blood-based biomarker for breast cancer diagnosis [20]. We found an overexpression of miR-191, a miRNA that has been detected up-regulated in formalin-fixed, paraffin-embedded breast cancer tissues [21], and breast cancer tissue [8,
22]. Additionally, there is evidence that demonstrates that miR-191 is deregulated at many diseases such as renal cell carcinoma [23], hepatocellular carcinoma [24], diabetes type 2 [25], inflammatory bowel disease [26], and breast cancer tissue [8,21,22]. Although our results show that miR-191 had the lowest $p$-value of the seven miRNAs in the analyzed BC serum, the use of miR191 recently proposed by $\mathrm{Hu}$ et al., as an endogenous control should be analyzed carefully [27]. Surprisingly miR-382 was found up-regulated with the highest $p$-value in our assay. Previous experiments done at our laboratory with Taqman low density arrays for breast cancer serum (unpublished data), demonstrated up-regulation of miR-382. We believe this is the first report of desregulation for miR-382 in breast cancer serum. Additionally, miR-382 had been found deregulated in massive macronodular adrenocortical disease and implicated in adrenocortical tumourigenesis [28], schizophrenia [29], osteosarcoma [30], and renal inner medullary interstitial fibrosis [31]. Finally, miR-382 appears to regulate the infectivity of HIV-1 in the target cells, and thus has an impact on HIV-1 latency [32].

We have determined the expression profile of seven miRNAs in a group of Mexican patients and provided strong evidence that the levels of breast cancerassociated miRNAs are significantly different from those in normal breast tissue. Moreover, we were able to distinguish between breast cancer and healthy individuals. The sensitivity and specificity for the seven miRNAs analyzed at optimal cutoff are $88.9 \%$ and $80 \%$ for miR-10b; $94 \%$ and $80 \%$ for miR-21; $88.9 \%$ and $80 \%$ for miR-125b; $94.4 \%$ and $100 \%$ for miR$145 ; 94.4 \%$ and $100 \%$ for miR-155; $72.2 \%$ and $90 \%$ for miR-191; and $94.4 \%$ and $90 \%$ for miR-382. Many studies have found a microRNA panel that accurately discriminates cancer from healthy subjects [22,33-35]. We analyzed a panel of three miRNAs (miR-145, miR145 and miR-382), and our results demonstrate that a combination of three miRNAs have better sensibility (97.6\%), specificity (100\%) and efficiency $(98 \%)$ that any other miRNA analyzed separately.

The number of papers reporting that circulating miRNAs could serve as noninvasive biomarkers for BC detection is increasing. Recently has been reported that miR-181a may represent a novel biomarker for primary BC as well as for early stage BC diagnosis [36], miR-125b expression is associated with breast cancer chemotherapeutic resistance [15]. We found that miR382 is associated with breast cancer and there is need for more research about the possible gene targets of this miRNA. Although we analyzed the serum levels of 
seven miRNAs in BC patients at different TNM stages, the number of early-stage BC samples was modest. In future investigations, we may be able to collect more early-stage BC samples to evaluate the role of serum miRNAs or other serum miRNAs associated with BC.

Many researchers are looking for effective bloodbased biomarkers for early BC detection. There is a need for prognostic and predictive markers for breast cancer to guide the selection of the most appropriate therapy for individual patients. Retrospective studies on many markers have been performed, but almost none were validated in prospective therapeutic trials or prospectively powered marker validation studies in the accurately selected patient population [37]. In recent years, the focus has been on circulating miRNAs, which have recently been reported to serve as an effective and non-invasive biomarker for detecting various cancers or other diseases [38-40]. Although the sensitivity and specificity of circulating miRNA biomarkers for BC is good in comparison with the serum biomarkers currently used, there is a long way to go before circulating miRNAs are used as a clinical diagnosis tool to detect $\mathrm{BC}$, because the levels of a circulating miRNA might be significantly higher or lower in various diseases. Future studies of circulating miRNA biomarkers may focus on combining the expression profiles of circulating miRNAs from all common diseases to obtain specific biomarkers for unique disease detection. Finally, it would be interesting to analyze the expression profile of these serum miRNA in a large number of breast cancer patients and healthy subjects looking for an early and non-invasive diagnostic tool.

In conclusion, we identified that seven serum miRNAs (miR-10b, miR-21, miR-125b, miR-145, miR155, miR-191 and miR-382) can potentially serve as novel noninvasive biomarkers for BC detection.

\section{Acknowledgments}

We would like to thank for financial support of the Teachers' Improvement Program (PROMEP) grants 103.5/07/2523 and 103.5/08/4285.

\section{References}

[1] Jemal A, Siegel R, Ward E, Hao Y, Xu J, et al. (2009) Cancer statistics, 2009. CA Cancer J Clin 59: 225-249.

[2] Calin GA, Croce CM (2006) MicroRNA signatures in human cancers. Nat Rev Cancer 6: 857-866.
[3] He L, Thomson JM, Hemann MT, Hernando-Monge E, Mu D, et al. (2005) A microRNA polycistron as a potential human oncogene. Nature 435: 828-833.

[4] Krutovskikh VA, Herceg Z (2010) Oncogenic microRNAs (OncomiRs) as a new class of cancer biomarkers. Bioessays 32: 894-904.

[5] Abba M, Mudduluru G, Allgayer H (2012) Micrornas In Cancer: Small Molecules, Big Chances. Anticancer Agents Med Chem. PMID: 2229-2749.

[6] Kosaka N, Iguchi H, Ochiya T (2010) Circulating microRNA in body fluid: A new potential biomarker for cancer diagnosis and prognosis. Cancer Sci 101: 2087-2092.

[7] Palma J, Yaddanapudi SC, Pigati L, Havens MA, Jeong S, et al. (2012) MicroRNAs are exported from malignant cells in customized particles. Nucleic Acids Res. doi: 10.1093/nar/ gks656.

[8] Iorio MV, Ferracin M, Liu CG, Veronese A, Spizzo R, et al. (2005) MicroRNA gene expression deregulation in human breast cancer. Cancer Res 65: 7065-7070.

[9] Roth C, Rack B, Muller V, Janni W, Pantel K, et al. (2010) Circulating microRNAs as blood-based markers for patients with primary and metastatic breast cancer. Breast Cancer Res 12: R90.

[10] Rothe F, Ignatiadis M, Chaboteaux C, Haibe-Kains B, Kheddoumi N, et al. (2011) Global microRNA expression profiling identifies MiR-210 associated with tumor proliferation, invasion and poor clinical outcome in breast cancer. PLoS One 6: e20980.

[11] Di Stefano V, Zaccagnini G, Capogrossi MC, Martelli F (2011) microRNAs as peripheral blood biomarkers of cardiovascular disease. Vascul Pharmacol 55: 111-118.

[12] Zhu W, Qin W, Atasoy U, Sauter ER (2009) Circulating microRNAs in breast cancer and healthy subjects. BMC Res Notes 2: 89 .

[13] Heneghan HM, Miller N, Kelly R, Newell J, Kerin MJ (2010) Systemic miRNA-195 differentiates breast cancer from other malignancies and is a potential biomarker for detecting noninvasive and early stage disease. Oncologist 15: 673-682.

[14] Asaga S, Kuo C, Nguyen T, Terpenning M, Giuliano AE, et al. (2011) Direct serum assay for microRNA-21 concentrations in early and advanced breast cancer. Clin Chem 57: 84-91.

[15] Wang H, Tan G, Dong L, Cheng L, Li K, et al. (2012) Circulating MiR-125b as a marker predicting chemoresistance in breast cancer. PLoS One 7: e34210.

[16] Bockmeyer CL, Christgen M, Muller M, Fischer S, Ahrens P, et al. (2011) MicroRNA profiles of healthy basal and luminal mammary epithelial cells are distinct and reflected in different breast cancer subtypes. Breast Cancer Res Treat 130: 735-745.

[17] Zou C, Xu Q, Mao F, Li D, Bian C, et al. (2012) MiR-145 inhibits tumor angiogenesis and growth by N-RAS and VEGF. Cell Cycle 11: 2137-2145.

[18] Sempere LF, Christensen M, Silahtaroglu A, Bak M, Heath CV, et al. (2007) Altered MicroRNA expression confined to specific epithelial cell subpopulations in breast cancer. Cancer Res 67: 11612-11620.

[19] Volinia S, Calin GA, Liu CG, Ambs S, Cimmino A, et al. (2006) A microRNA expression signature of human solid tumors defines cancer gene targets. Proc Natl Acad Sci U S A 103: 2257-2261.

[20] Wang F, Zheng Z, Guo J, Ding X (2010) Correlation and quantitation of microRNA aberrant expression in tissues and sera from patients with breast tumor. Gynecol Oncol 119: 586-593. 
[21] Hui AB, Shi W, Boutros PC, Miller N, Pintilie M, et al. (2009) Robust global micro-RNA profiling with formalinfixed paraffin-embedded breast cancer tissues. Lab Invest 89: 597-606.

[22] Mar-Aguilar F, Luna-Aguirre CM, Moreno-Rocha JC, AraizaChávez J, Trevino V, et al. (2012) Differential expression of miR-21, miR-125b and miR-191 in breast cancer tissue. Asia-Pac J Clin Oncol 1-7. doi: 10.1111/j.1743-7563.2012. 01548.x.

[23] Hauser S, Wulfken LM, Holdenrieder S, Moritz R, Ohlmann $\mathrm{CH}$, et al. (2012) Analysis of serum microRNAs (miR-26a-2*, miR-191, miR-337-3p and miR-378) as potential biomarkers in renal cell carcinoma. Cancer Epidemiol 36: 391-394.

[24] Elyakim E, Sitbon E, Faerman A, Tabak S, Montia E, et al. (2010) hsa-miR-191 is a candidate oncogene target for hepatocellular carcinoma therapy. Cancer Res 70: 8077-8087.

[25] Zampetaki A, Kiechl S, Drozdov I, Willeit P, Mayr U, et al. (2010) Plasma microRNA profiling reveals loss of endothelial miR-126 and other microRNAs in type 2 diabetes. Circ Res 107: 810-817.

[26] Paraskevi A, Theodoropoulos G, Papaconstantinou I, Mantzaris G, Nikiteas N, et al. (2012) Circulating MicroRNA in inflammatory bowel disease. J Crohns Colitis 9: 900-4.

[27] Hu Z, Dong J, Wang LE, Ma H, Liu J, et al. (2012) Serum microRNA profiling and breast cancer risk: The use of miR484/191 as endogenous controls. Carcinogenesis 33: 828-834.

[28] Bimpaki EI, Iliopoulos D, Moraitis A, Stratakis CA (2010) MicroRNA signature in massive macronodular adrenocortical disease and implications for adrenocortical tumourigenesis. Clin Endocrinol (Oxf) 72: 744-751.

[29] Santarelli DM, Beveridge NJ, Tooney PA, Cairns MJ (2011) Upregulation of dicer and microRNA expression in the dorsolateral prefrontal cortex Brodmann area 46 in schizophrenia. Biol Psychiatry 69: 180-187.
[30] Thayanithy V, Sarver AL, Kartha RV, Li L, Angstadt AY, et al. (2012) Perturbation of 14q32 miRNAs-cMYC gene network in osteosarcoma. Bone 50: 171-181.

[31] Kriegel AJ, Liu Y, Cohen B, Usa K, Liang M (2012) MiR-382 targeting of kallikrein 5 contributes to renal inner medullary interstitial fibrosis. Physiol Genomics 44: 259-267.

[32] Purohit V, Rapaka RS, Rutter J, Shurtleff D (2012) Do Opioids Activate Latent HIV-1 by Down-Regulating Anti-HIV microRNAs? J Neuroimmune Pharmacol 7: 519-523.

[33] Wang S, Wang L, Bayaxi N, Li J, Verhaegh W, et al. (2012) A microRNA panel to discriminate carcinomas from high-grade intraepithelial neoplasms in colonoscopy biopsy tissue. Gut. doi:10.1136/gutjnl-2011-301554.

[34] Song MY, Pan KF, Su HJ, Zhang L, Ma JL, et al. (2012) Identification of serum microRNAs as novel non-invasive biomarkers for early detection of gastric cancer. PLoS One 7: e33608.

[35] Chen ZH, Zhang GL, Li HR, Luo JD, Li ZX, et al. (2012) A panel of five circulating microRNAs as potential biomarkers for prostate cancer. Prostate 72: 1443-52.

[36] Guo LJ, Zhang QY (2012) Decreased serum miR-181a is a potential new tool for breast cancer screening. Int J Mol Med 30: $680-686$

[37] Kaufmann M, Pusztai L (2011) Use of standard markers and incorporation of molecular markers into breast cancer therapy: Consensus recommendations from an International Expert Panel. Cancer 117: 1575-1582.

[38] Weiland M, Gao XH, Zhou L, Mi QS (2012) Small RNAs have a large impact: Circulating microRNAs as biomarkers for human diseases. RNA Biol 9: 850-859.

[39] Cortez MA, Welsh JW, Calin GA (2012) Circulating microRNAs as noninvasive biomarkers in breast cancer. Recent Results Cancer Res 195: 151-161.

[40] Creemers EE, Tijsen AJ, Pinto YM (2012) Circulating microRNAs: novel biomarkers and extracellular communicators in cardiovascular disease? Circ Res 110: 483-495. 


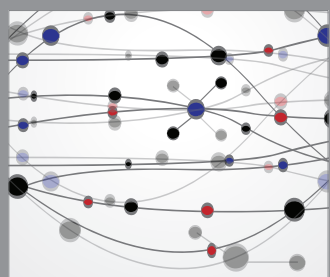

The Scientific World Journal
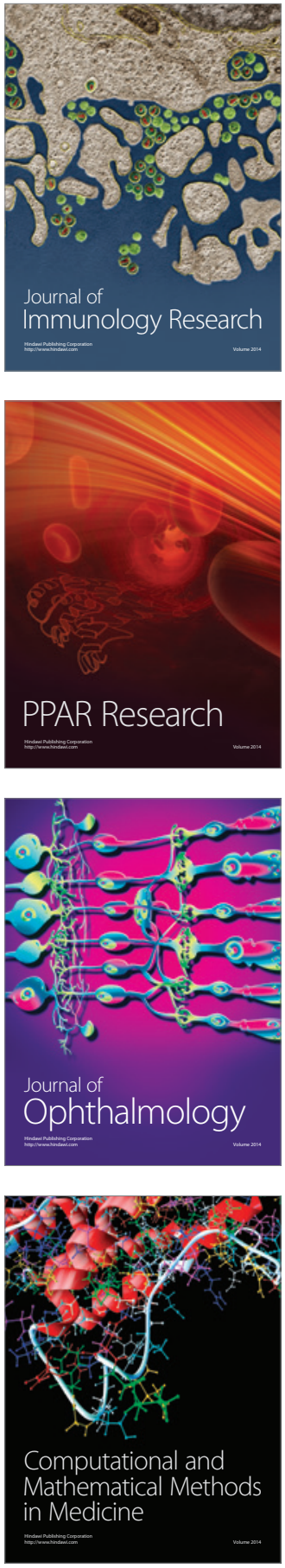

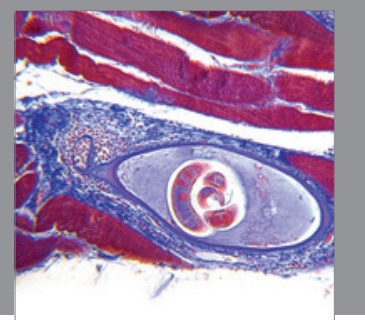

Gastroenterology

Research and Practice
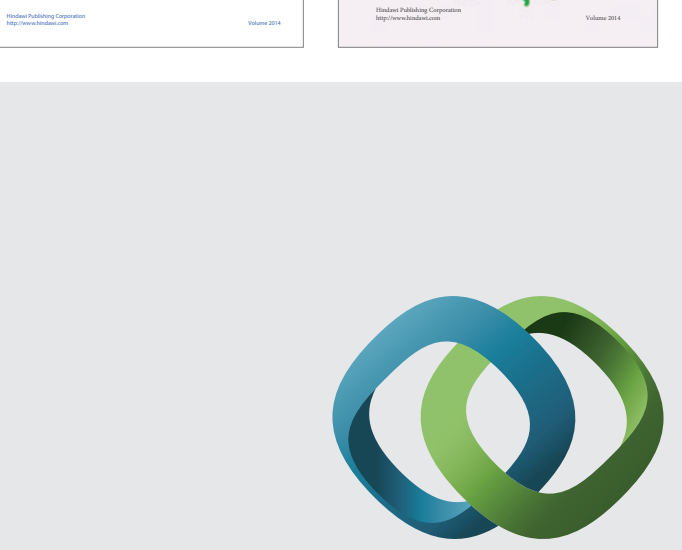

\section{Hindawi}

Submit your manuscripts at

http://www.hindawi.com
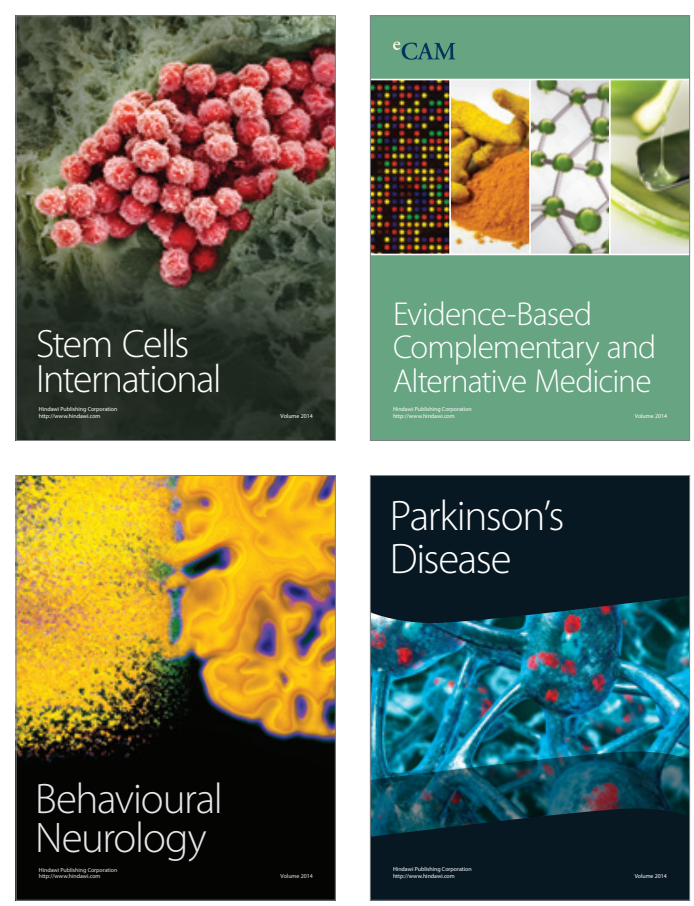

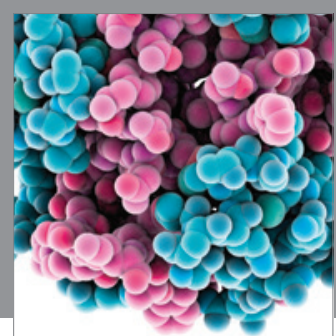

Journal of
Diabetes Research

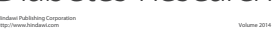

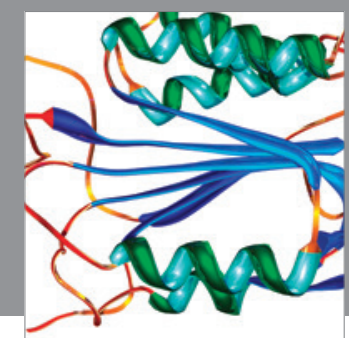

Disease Markers
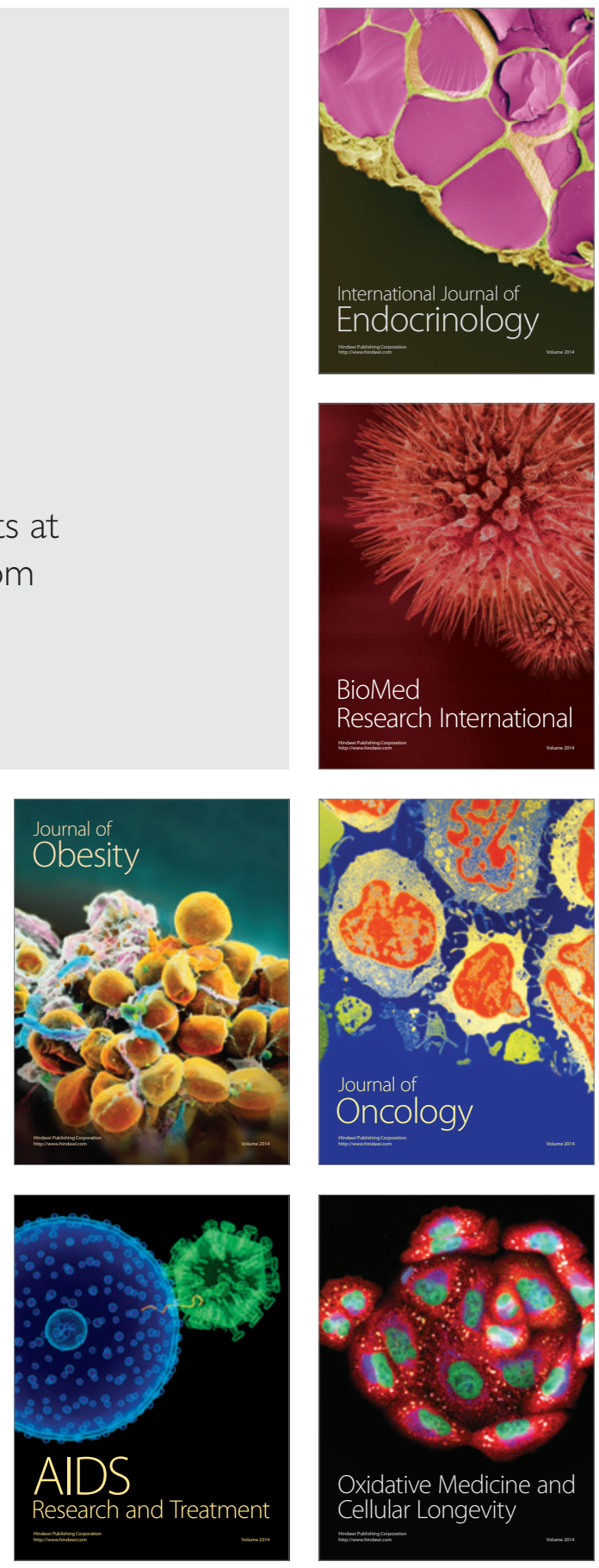